\title{
The European LEMS Registry: Baseline Demographics and Treatment Approaches
}

\author{
Renato Mantegazza (D) Andreas Meisel · Joern P. Sieb • \\ Gwendal Le Masson · Claude Desnuelle · Mirko Essing
}

To view enhanced content go to www.neurologytherapy-open.com

Received: July 13, 2015 / Published online: November 2, 2015

(C) The Author(s) 2015. This article is published with open access at Springerlink.com

\section{ABSTRACT}

Introduction: Lambert-Eaton myasthenic syndrome (LEMS) is a rare autoimmune disorder affecting the neuromuscular junction, clinically characterized by proximal muscle weakness and autonomic changes. LEMS is often associated with an underlying tumor (paraneoplastic form) but also occurs in the

Electronic supplementary material The online version of this article (doi:10.1007/s40120-015-0034-0) contains supplementary material, which is available to authorized users.

R. Mantegazza $(\square)$

Istituto Neurologico Carlo Besta, Milan, Italy

e-mail: rmantegazza@istituto-besta.it;

renato.mantegazza@istituto-besta.it

A. Meisel

Charité Universitätsmedizin Berlin, Berlin,

Germany

J. P. Sieb

Hanse-Klinikum Stralsund, Stralsund, Germany

G. Le Masson

Hôpital Pellegrin, Bordeaux, France

C. Desnuelle

Centre de Référence Maladies

Neuromusculaires-CHU de Nice, Nice, France

M. Essing

BioMarin Europe, Ltd., London, UK absence of cancer (idiopathic form). Treatment consists of immunomodulation (immunosuppression), anticancer treatment when carcinoma is present, and symptomatic treatment [acetylcholinesterase inhibitors and potassium channel blockers, e.g., amifampridine (3,4-diaminopyridine, i.e., 3,4-DAP), to improve neurotransmission]. Although there has long been information from case reports, several randomized controlled trials, and treatment guidelines, population data are still scarce.

Methods: The LEMS patient registry was launched in the European community in mid-2010 as a voluntary, multinational, observational, non-interventional program to collect structured empirical data on clinical course, treatment utilization, and safety and efficacy from the use of LEMS-specific treatments. Results: Sixty-nine patients have been enrolled [36 males, 32 females, 1 gender not reported; mean age 61.5 (27-84) years]. Eighteen patients (26\%) were diagnosed with an associated carcinoma. At the time of enrollment, the majority of patients (65\%) were receiving amifampridine [either compounded 3,4-DAP (22\%) or 3,4-DAP phosphate, Firdapse $\left.{ }^{\circledR}(43 \%)\right]$. 
At enrollment, most patients demonstrate a profile of mild-to-moderate deficits in daily functioning but generally have good muscle strength, albeit with reduced deep tendon reflexes, frequent ataxia during walking, and signs of autonomic dysfunction including dry mouth, bladder dysfunction, and constipation. Conclusion: The LEMS European Union registry will continue to enroll patients and periodically report the accrued longitudinal data obtained on clinical assessments and laboratory findings, treatment practices, the safety and efficacy of treatment approaches, and long-term clinical outcomes.

Funding: BioMarin Pharmaceutical Inc., Novato, CA, USA.

Keywords: 3,4-Diaminopyridine (3,4-DAP); Amifampridine; Firdapse; Lambert-Eaton; LEMS; Registry

\section{INTRODUCTION}

Lambert-Eaton myasthenic syndrome (LEMS) is a rare autoimmune disorder caused by pathogenic autoantibodies directed against presynaptic voltage-gated $\mathrm{Ca}_{\mathrm{v}} 2.1$ (P/Q type) calcium ion channel (VGCC) at the neuromuscular junction (NMJ) [1, 2]. It is clinically characterized by limb girdle weakness, increased fatigability, depressed tendon reflexes, and autonomic changes. The decreased calcium influx prevents sufficient release of acetylcholine (ACh) from the nerve terminals, subsequent stimulation of the post-synaptic membrane, and attenuates normal muscle contraction [3-5].

LEMS is estimated to affect 1:100,000 people in the European community [6] with an incidence of 0.48 to 0.75 per million [7]. In a study from South Holland, Wirtz et al. [7] found a LEMS prevalence of 2.3 per million and an annual incidence rate of 0.5 per million. The true incidence of LEMS is unknown. LEMS is reported to be associated with cancer (paraneoplastic form) in more than 50\% of cases, with small cell lung cancer (SCLC) being the most frequently observed associated cancer. The reported percentage of newly diagnosed LEMS cases associated with small SCLC ranges from $40 \%$ to $70 \%$ [7-9]. The prevalence of LEMS in patients with SCLC is estimated to be approximately 3\% [10]. The low prevalence relative to incidence reflects the poor survival of LEMS patients with the paraneoplastic type of disease, and specifically the poor survival of patients with SCLC. The clinical manifestations of LEMS often precede the detection of an underlying carcinoma. The associated cancer is usually discovered within the first 2 years after the onset of LEMS and, in almost all cases, within 4 years [11]. LEMS is diagnosed based on the often suggestive clinical presentation, electrophysiological studies, testing for $\mathrm{Ca}_{\mathrm{v}} 2.1$ P/Q-type VGCC autoantibodies, and through clinical evaluation (diagnostic considerations are described further in the "Discussion").

Besides the muscular weakness, LEMS is associated with signs of autonomic dysfunction such as dry mouth, constipation, and erectile dysfunction. Activities associated with daily functioning, e.g., climbing stairs without support, rising from a low sitting or squatting position, cycling, are typically affected mildly or moderately [12]. The onset of symptoms, typically at $\geq 40$ years of age in patients with cancer (median age 60 years), and between 20 and 50 years of age in those without (median age 35 years), is usually gradual and insidious [13]. Rarely, respiratory function may be impaired to the extent of requiring mechanical ventilation. 


\section{LEMS EU Registry: Background and Objectives}

The European Federation of Neurological Societies (EFNS) has published guidelines from two different task forces [14-16]. These guidelines recommend treatment based on consensus assessments of all available evidence, although nearly all of the published empirical evidence consists of disperse case reports and a handful of randomized controlled trials. There is little information on the population statistics of this disorder or data on the implementation of treatment practices, nor is there comprehensive information on the long-term efficacy, safety, and clinical outcomes of treatments.

Key objectives of the registry are to: (1) obtain observational safety data on patients diagnosed with LEMS, (2) monitor for safety signals in patients treated with Firdapse ${ }^{\circledR}$ (BioMarin Europe Limited, London, UK), the potassium channel blocker phosphate salt form of 3,4-diaminopyridine (3,4-DAP; amifampridine phosphate) approved in December 2009 by the European Commission for the symptomatic treatment of LEMS, (3) gather the same observational information from patients with LEMS who are not treated with Firdapse to better understand the background incidences and enable inter-treatment comparisons of different therapies, and (4) collect additional data on the long-term treatment practices and safety and efficacy of LEMS-specific treatments and other treatments prescribed in this population.

The purpose of the present article is to provide a first interim report on the demographics and clinical characteristics of the accrued patient population at the time of baseline enrollment into the registry. Follow-up data for patients will continue to be accrued and will be reported at a later date. The registry is sponsored by BioMarin Pharmaceutical Inc. (Novato, CA, USA).

\section{METHODS}

\section{Registry Design, Patient Enrollment, and Data Reporting}

The LEMS data registry was started as a voluntary multi-center, multinational, observational program for patients who have been diagnosed with LEMS to enable a better characterization of its natural history. Enrollment centers are only in regions where regulatory approval for marketing of Firdapse has been granted, i.e., in the European Union (EU) and in Switzerland. Within these countries, the registry was planned based on where the largest patient populations have been reported. Patients currently participating in sponsored trials of Firdapse, however, are excluded from participation. Beyond these general requirements for eligibility, the registry is voluntary and there is no obligation that patients be enrolled in marketing approved countries.

The first patient was enrolled into the LEMS EU registry on May 5, 2010. The registry will continue for 5 years following initial patient enrollment and then will continue to obtain follow-up information for 3 years after the last patient is enrolled, or until 2018. The registry is non-interventional and no experimental treatments or assessments are involved. All patients with a confirmed diagnosis of LEMS disease and who provide informed consent are eligible to participate in this program. Each enrolled patient is assigned a unique subject identifier. The protocol, patient information sheet, and consent form have been approved by ethics committees, subject to all applicable 
local laws. All procedures followed were in accordance with the ethical standards of the responsible committee on human experimentation (institutional and national) and in accordance with the Helsinki Declaration of 1964, as revised in 2013.

\section{Data Collection and Entry}

The registry relies on voluntary submission of data by participating clinical investigators. Data from 29 EU centers located in 4 countries: Germany (10 centers), Italy (4 centers), France (13 centers), and Spain ( 2 centers) are collected and tabulated. Specific clinical and laboratory assessments collected during participation in the registry are further described below. Assessment data are continually entered by clinical sites into a centralized registry database via a validated web-portal application.

\section{Classification and Tracking of LEMS-Specific and LEMS-Related Treatments}

Treatment information was collected at the time of patient enrollment. Due to the observational, non-interventional nature of the LEMS registry, patients may have been newly diagnosed with LEMS at the time of enrollment or may have been previously diagnosed and treated for varying lengths of time. Treatment information was organized to reflect that patients could be on different LEMS treatments at different times, both before and/or after the time of enrollment, and all changes in treatments (drug, dose, start/ stop) were made at the discretion of the treating physician and this information was recorded in the registry database.

LEMS treatments are classified into 1 of 4 categories: (1) Firdapse (Firdapse), (2) 3,4-DAP (base), (3) other LEMS treatments (Other
Treatments) and, (4) unknown treatments (Unknown Treatments). Further, LEMS-specific treatments refer to Firdapse, 3,4-DAP as the base form, or other LEMS treatments that include, but are not limited to, guanidine, acetylcholinesterase (AChE) inhibitors, prednisone, azathioprine, cyclosporine, intravenous immunoglobulin (IVIg), and plasmapheresis. A treatment was classified as an Unknown Treatment when it was known that a patient was receiving LEMS-specific treatment(s), but this datum was not available from the clinical site at the time of the interim report.

\section{Clinical and Laboratory Assessments}

A panel of international clinical experts in the care of patients with LEMS was convened to recommend a set of assessments appropriate for the long-term monitoring of clinical status and outcomes for this patient population (Table 1). In addition to their use in establishing an initial diagnosis, the recommended assessments were chosen to monitor changes in LEMS-related clinical manifestations and to stage any disease progression across the life-long course of the disease. Key assessments for LEMS are to periodically obtain: electrophysiological data, quantitative myasthenia gravis (QMG) scores, assessments of muscle strength, testing for presence and severity of ataxia, electrocardiographic (ECG) data (specifically to evaluate QT interval abnormalities), pulmonary function tests (PFTs), and self-report questionnaires on daily functioning and general health status. Each patient's treating physician determined the actual timing and frequency of each assessment according to individualized needs.

If the time of initial diagnosis of LEMS did not approximately coincide with the time of 
Table 1 Annual recommended annual clinical assessments for management of Lambert-Eaton myasthenic syndrome

\begin{tabular}{|c|c|}
\hline Assessment & Test/assessment parameters \\
\hline Electrophysiology & CMAP amplitude; \% increment; \% decrement \\
\hline $\begin{array}{l}\text { Antibody assessment } \\
\qquad(\operatorname{IgG})\end{array}$ & VGCC antibody titer [anti-Ca $a_{v} 2.1$ (P/Q-type) VGCC] \\
\hline QMG & QMG Total Score \\
\hline Muscle strength & $\begin{array}{l}\text { Triceps, wrist flexion, wrist extension, hamstring, quadriceps, foot flexor, foot extensor, iliopsoas, } \\
\text { gluteus maximus, neck flexor, neck extensor, and facial muscles }\end{array}$ \\
\hline Reflexes & Knee, achilles, biceps, triceps \\
\hline Ataxia assessment & Line walk test, Romberg's test, left and right finger-to-nose, left and right heel-to-knee tests \\
\hline $\begin{array}{l}\text { Autonomic nervous } \\
\text { system }\end{array}$ & $\begin{array}{l}\text { Dry eyes, dry mouth, pupil reflex, erectile dysfunction/impotence, constipation, bladder function, } \\
\text { and orthostatic intolerance }\end{array}$ \\
\hline $\begin{array}{l}\text { Electrocardiography } \\
\text { (ECG) }\end{array}$ & HR, PQ Interval, PR Interval, QT Interval, QTcB Interval, QTcF Interval, QRS Interval \\
\hline Spirometry & $\begin{array}{l}\text { 1-s forced expiratory volume }\left(\mathrm{FEV}_{1}\right) \text {, forced vital capacity }(\mathrm{FVC}) \text {, total lung capacity (TLC), } \\
\text { maximum voluntary ventilation }(\mathrm{MVV})\end{array}$ \\
\hline Daily functioning & Walking ability, cycling, getting up from sitting and squatting, climbing upstairs \\
\hline Health status & EuroQol EQ-5D \\
\hline
\end{tabular}

$C M A P$ compound muscle action potential, $I g G$ immunoglobulin $\mathrm{G}, Q M G$ quantitative myasthenia gravis, $V G C C$ voltage-gated calcium ion channel

enrollment into the registry (many patients having been previously diagnosed and already receiving treatment for varying periods of time), a particular assessment may not have been performed or available until the next scheduled follow-up visit and was classified as "Missing" for the purposes of data analysis.

Electrophysiological studies were performed according to a standardized assessment protocol specifically for the evaluation of LEMS $[17,18]$. Compound muscle action potential (CMAP) amplitude obtained from electromyography (EMG) is measured in $\mathrm{mV}$ and reflects the severity of neuromuscular block [19]. Following repetitive nerve stimulation (RNS) at rates of 3-5 Hz, a decremental response expressed as a percentage of up to $8 \%$ in normal muscles is normally seen. In contrast, the CMAP amplitude in resting muscle in patients with LEMS characteristically shows a decrementing pattern of greater than $10 \%$ in the 4 th or 5 th amplitude response following RNS. Even a decrement of 5\% may be abnormal if artifacts, a common accompaniment to electrophysiological testing, have been ruled out as a cause of the change. An additional electrophysiologic characteristic of LEMS is a marked and brief percent increment in CMAP amplitude ( $>60 \%$ ) after a maximal voluntary contraction by the patient relative to the resting state.

QMG symptoms were assessed by standardized protocol $[20,21]$. Since assessments are occasionally not performed by investigators for one or more of the 13 QMG sub-scales, an alternative estimate of the QMG Total Score (the '\%Standardized QMG Total Score') is computed using direct standardization of the available assessment 
data to permit improved comparability of mean values and other descriptive statistics between treatment groups. In these cases, each patient's raw QMG Total Score is standardized on the basis of the number of sub-scales for which assessment data are available: \%Standardized QMG Total Score $=[$ raw QMG Total Score $/(N$ of sub-scales with available data $\times$ maximum sub-scale score of 3)]. The \%Standardized QMG Total Score is interpreted for the purposes of this registry as the percent of the maximum possible Total QMG Score of 39 when a complete assessment has been performed (e.g., a \%Standardized QMG Total Score of 0.66 or $66 \%$ implies that a patient's Total QMG Score was equivalent to 26 on a complete QMG assessment).

Patients' muscle strength is assessed in 13 muscles or muscle groups: biceps, triceps, wrist flexion, wrist extension, hamstring, quadriceps, foot flexor, foot extensor, iliopsoas, gluteus maximus, neck flexor, neck extensor, and facial muscles. Muscle strength is rated on a 5-point scale for each muscle group, ranging from 0 (absent voluntary contraction) to 5 (full strength). The strength of facial muscles is rated as: 1 (normal), 2 (abnormal), or 3 (not done).

Deep tendon reflexes are assessed at the knee, Achilles, biceps, and triceps. Ataxia is assessed from a line walk test, Romberg's test, and finger-to-nose and heel-to-knee tests. Any signs of autonomic dysfunction are recorded (i.e., dry eyes or mouth, abnormal pupil reflexes, constipation, bladder dysfunction, erectile dysfunction/impotence, orthostatic intolerance).

Although there are very few reports of adverse cardiovascular effects (serious events have been reported in only 2 cases [22, 23] ), 12-lead ECGs are performed to further evaluate and ensure safety. The ECG is also specifically evaluated for QT-prolongation and other interval abnormalities (i.e., PR, QTcB, QTcF, QRS).

Because of the frequent association of LEMS with SCLC, and published reports have cited involvement of respiratory muscles and the diaphragm in LEMS [24], spirometry is recommended periodically if there are concerns about a patient's respiratory functioning or to monitor disease progression. Forced vital capacity (FVC) should be assessed at regular intervals to detect and evaluate the extent of any progressive worsening of muscular weakness. PFTs were performed for registry patients according to standard pulmonary testing practices, and percent of predicted values relative to normal subjects were derived, when possible, for key parameters [forced expiratory volume in $1 \mathrm{~s}$ $\left(\mathrm{FEV}_{1}\right)$ and $\left.\mathrm{FVC}\right]$ using published normative data [25], taking into account patient's sex, age, and height.

Information on health status was obtained periodically using the EuroQoL 5D (EQ-5D), a widely employed health status questionnaire [26]. The EuroQoL 5D assesses an individual's daily functioning via an Investigator's rating of a patient's ability to perform each of 12 activities as either normal/as expected or reduced/limited.

The evaluation of treatment safety will appear in future reports and will include analyses of adverse events, concomitant medications, and clinical laboratory tests.

\section{Data Analysis}

The database used for analysis consisted of all available data for any assessments that were performed in association with registry enrollment. Clinical data were tabulated according to which of the 4 treatments a patient was receiving at the time of consent 
and enrollment into the registry (Firdapse, 3,4-DAP, Other Treatment, or Unknown Treatment). Patients receiving Firdapse at enrollment, either alone or in combination with any other LEMS-specific treatment, were classified into the Firdapse group. Similarly, patients receiving 3,4-DAP at enrollment, either alone or in combination with any other LEMS-specific treatment, were classified into the 3,4-DAP group. Patients who were not receiving 3,4-DAP or Firdapse at enrollment and who were either known not to be receiving treatment or were not receiving any other LEMS treatment, were classified into the Other Treatments group. Patients whose treatment status at enrollment was unknown were classified into the Unknown Treatments group.

Assessment data are summarized using descriptive methods only using means, standard deviations (SD), medians, quartiles, and ranges (min, max). Due to the observational and non-interventional nature of the registry program, no attempts will be made to perform inferential statistical evaluation. When statistical data are presented as pooled data, data will be combined from all available patients in the four treatment groups.

\section{RESULTS}

\section{Registry Population}

A total of 69 patients have been enrolled into the LEMS registry thus far (Germany $n=30$; Spain $n=0$; Italy $n=22$; France $n=17$ ). At time of enrollment, patients were receiving Firdapse $(n=30), 3,4$-DAP $(n=15)$, Other Treatments $(n=16)$, or Unknown Treatments $(n=8)$.

Four patients have been discontinued from the registry. Three patients, all who had received 3,4-DAP, were treated as discontinued from the registry due to death. Two deaths were the result of cardiac arrest. The third death was the result of renal failure followed by multi-organ failure. In all 3 cases, death was reported as unrelated to 3,4-DAP and unrelated to other LEMS medications. Another patient who had also received 3,4-DAP withdrew from the registry due to moving to another city and has been lost to follow-up.

\section{Population Demographics and Other Characteristics}

Table 2 summarizes patient demographics and other characteristics by treatment group (data were not available for one enrolled patient). The mean age at time of enrollment is 61.5 years (SD 11.9 years, range $27-84$ years). There are several more males $(36 / 69 ; 52.2 \%)$ than females $(32 / 69$; $46.4 \%)$ and the majority of patients are Caucasian (51/69; 73.9\%). Information on ethnicity was not available from patients enrolled at centers in France due to national reporting restrictions prohibiting the collection of ethnicity data. Most patients either never smoked $(32 / 69 ; 46.4 \%)$ or smoked previously but have since quit $(25 / 69 ; 36.2 \%)$, and 10 patients (14.5\%) are current smokers. Two patients have unreported information on smoking history.

Results for $\mathrm{Ca}_{\mathrm{v}} 2.1 \mathrm{P} / \mathrm{Q}$-type VGCC antibody assays were positive (+ve) for 19 of the 21 patients for whom VGCC data were available in the overall registry population, with associated titers ranging from 32.0 to $735.0 \mathrm{pmol}$ per liter (pmol/L) (mean $302.9 \mathrm{pmol} / \mathrm{L}, \quad$ SD 249.87; normal range $<20 \mathrm{pmol} / \mathrm{L}$ [27]).

At the time of enrollment, the majority of patients were receiving either Firdapse (30/69; $43.5 \%)$ or 3,4 -DAP $(15 / 69 ; 21.7 \%)$. Eleven patients were diagnosed with lung cancer (Firdapse $=4, \quad 3,4-\mathrm{DAP}=2, \quad$ Unknown 
Table 2 Registry population demographics and baseline characteristics

\begin{tabular}{|c|c|c|c|c|c|}
\hline \multirow[t]{2}{*}{ Characteristic } & \multicolumn{4}{|c|}{ Treatment group (treatment received at time of enrollment) } & \multirow{2}{*}{ Total $(N=69)$} \\
\hline & Firdapse $(n=30)$ & 3,4-DAP $(n=15)$ & Other $(n=16)$ & Unknown $(n=8)$ & \\
\hline \multicolumn{6}{|c|}{ Age at time of informed consent (years) } \\
\hline$n$ & 30 & 15 & 16 & 6 & 67 \\
\hline Mean (SD) & $60.5(10.1)$ & $64.5(12.4)$ & $63.7(11.9)$ & $52.8(16.7)$ & $61.5(11.9)$ \\
\hline Min-max & $38.0-77.0$ & $38.0-84.0$ & $34.0-76.0$ & $27.0-74.0$ & $27.0-84.0$ \\
\hline \multicolumn{6}{|l|}{ Gender, $n(\%)$} \\
\hline Male & $16(53.3)$ & $7(46.7)$ & $8(50.0)$ & $5(62.5)$ & $36(52.2)$ \\
\hline Female & $14(46.7)$ & $8(53.3)$ & $8(50.0)$ & $2(25.0)$ & $32(46.4)$ \\
\hline Unreported & & & & $1(12.5)$ & $1(1.4)$ \\
\hline \multicolumn{6}{|c|}{ Smoking history, $n(\%)$} \\
\hline Current smoker & $6(20.0)$ & $0(0.0)$ & $3(18.8)$ & $1(12.5)$ & $10(14.5)$ \\
\hline Smoked previously & $12(40.0)$ & $2(13.3)$ & $6(37.5)$ & $5(62.5)$ & $25(36.2)$ \\
\hline Never smoked & $12(40.0)$ & $13(86.7)$ & $6(37.5)$ & $1(12.5)$ & $32(46.4)$ \\
\hline Unreported & & & $1(6.3)$ & $1(12.5)$ & $2(2.9)$ \\
\hline \multicolumn{6}{|c|}{ Pack-years for current smokers ${ }^{\mathrm{b}}$} \\
\hline$n$ & 6 & 0 & 3 & 1 & 10 \\
\hline Mean (SD) & $23.0(19.2)$ & & $22.0(22.3)$ & $30.0^{\mathrm{a}}$ & $23.4(17.9)$ \\
\hline Min-max & $4.0-60.0$ & & $4.0-47.0$ & $30.0-30.0$ & $4.0-60.0$ \\
\hline \multicolumn{6}{|c|}{ Pack-years for previous smokers ${ }^{b}$} \\
\hline$n$ & 11 & 2 & 6 & 5 & 24 \\
\hline Mean (SD) & $27.0(24.7)$ & $45.0(21.2)$ & $29.0(28.8)$ & $95.4(146.0)$ & $43.3(70.3)$ \\
\hline Min-max & $4.0-82.0$ & $30.0-60.0$ & $1.0-80.0$ & $16.0-356.0$ & $1.0-356.0$ \\
\hline
\end{tabular}

3,4-DAP 3,4-diaminopyridine, $S D$ standard deviation

a $\mathrm{SD}$ not computed

b Pack-years computed as: [(average number of cigarettes per day/20) $\times$ number of years smoked]

Treatment $=3$, and Other Treatment $=2$ ). A patient in the Firdapse treatment group with SCLC was also diagnosed with cervical carcinoma. Six other patients were diagnosed with other forms of cancer: adenocarcinoma of the lower oesophagus and epidermoid carcinoma of the vocal chords (Firdapse), breast neoplasia (Firdapse), kidney cancer and metastasized colon cancer with pulmonary and hepatic metastasis (Other Treatment), malignant thymoma with associated myasthenia gravis (Other Treatment), Merkel cell carcinoma with concomitant pneumopathy (Firdapse), and multiple myeloma (3,4-DAP).

\section{Treatment Characteristics}

A total of 30 patients are enrolled in the Firdapse treatment group. Most of these patients had been receiving Firdapse for a 
period of between 2 days and up to 14.8 years at the time of enrollment into the registry. At least 16 of these patients had been on Firdapse for $\geq 1$ year prior to registry enrollment. Patients were receiving total daily doses of $10-80 \mathrm{mg}$. A total of 40 patients in the registry have received Firdapse at some point, either before, during, and/or after enrollment. It is up to the prescribing physician's judgment what the appropriate risk-benefit relationship should be for an individual patient in determining the appropriate dose of Firdapse if it is prescribed outside of the recommended guidelines. Fifteen patients were receiving 3,4-DAP base at the time of enrollment with total daily doses of 20-90 mg. Most patients had been receiving LEMS-specific or LEMS-related treatment(s) for varying periods of time at the time of enrollment into the registry.

Table 3 summarizes LEMS-specific treatments other than Firdapse or 3,4-DAP base that were received at any time (i.e., received prior to or after registry enrollment) by treatment group.
There were no other LEMS-specific treatments received by patients in the Unknown LEMS treatments group. Besides Firdapse or 3,4-DAP, immunomodulators, most commonly immunosuppressants, were the most frequently employed LEMS-specific treatment: 12 patients received azathioprine, 2 patients received cyclophosphamide, 4 patients received mycophenolate mofetil (MMF), 2 patients received cyclosporine A, 19 patients received prednisone, 1 patient received methylprednisolone, and 10 patients received IVIg therapy. Twenty-two patients received pyridostigmine.

Twenty-six patients $(37.7 \% ; 26 / 69)$ had only ever received monotherapy, consisting of Firdapse (17.4\%; 12/69), 3,4-DAP base (13.0\%; $9 / 69)$, pyridostigmine $(2.9 \% ; 2 / 69)$, prednisone $(2.9 \% ; 2 / 69)$, and normal immunoglobulin (1.4\%; $1 / 69)$. The remaining patients $(62.3 \%$; $43 / 69$ ) were on LEMS polytherapy treatments consisting of any combination of LEMS medications, received either concomitantly

Table 3 LEMS-specific treatments administered at any time, by drug class and registry treatment group

\begin{tabular}{lllll}
\hline Drug class & \multicolumn{4}{l}{ Treatment group (LEMS-specific treatment received at time of enrollment) } \\
\cline { 2 - 5 } & Firdapse $(\boldsymbol{n}=\mathbf{3 0})$ & $\mathbf{3 , 4 - D A P}(\boldsymbol{n}=\mathbf{1 5})$ & Other $(\boldsymbol{n}=\mathbf{1 6})$ & Unknown $(\boldsymbol{n}=\mathbf{8})$ \\
\hline Immunomodulation & & & & \\
Azathioprine & $6(20.0)$ & $2(13.3)$ & $4(25.0)$ & $0(0.0)$ \\
Cyclophosphamide & $0(0.0)$ & $0(0.0)$ & $2(12.5)$ & $0(0.0)$ \\
Mycophenolate mofetil & $1(3.3)$ & $2(13.3)$ & $1(6.3)$ & $0(0.0)$ \\
Cyclosporine A & $2(6.7)$ & $0(0.0)$ & $0(0.0)$ & $0(0.0)$ \\
Prednisone & $8(26.7)$ & $1(6.7)$ & $10(62.5)$ & $0(0.0)$ \\
Methylprednisolone & $0(0.0)$ & $1(6.7)$ & $0(0.0)$ & $0(0.0)$ \\
Immunoglobulin & $3(10.0)$ & $2(13.3)$ & $5(31.3)$ & $0(0.0)$ \\
Parasympathomimetics & $11(36.7)$ & $5(33.3)$ & $6(37.5)$ & $0(0.0)$ \\
\hline
\end{tabular}

Values in parentheses are expressed in percentage

3,4-DAP 3,4-diaminopyridine, LEMS Lambert-Eaton myasthenic syndrome

Counts of treatments are not mutually exclusive; patients could have received a LEMS-specific treatment as a monotherapy or could have received several LEMS-specific treatments as part of combination therapy 
and/or individually but at different times. In addition, for most patients receiving polytherapy, changes and adjustments in the specific combinations of treatments administered, doses, and durations were commonplace over the clinical course of individual patients.

\section{Diagnostic Characteristics and Clinical Status of Patients at Enrollment}

The CMAP amplitude parameter assessed during EMG evaluation, along with autoantibody testing $\left(\mathrm{Ca}_{\mathrm{v}} 2.1 \mathrm{P} / \mathrm{Q}\right.$-type VGCC) and a clinical evaluation of symptoms, were the primary methods used to confirm the diagnosis of LEMS.

Results were positive (+ve) at baseline for 19 of the 21 patients for whom VGCC data were available in the overall registry population. Associated titers ranged from 32.0 to 735.0 pmol/L (mean 302.9 pmol/L, SD 249.87).

\section{Electrophysiology}

The mean percent increment and decrement values and the individual values for the patients for whom EMG testing was available near the time ( \pm 1 month) of enrollment into the registry indicated abnormality in neuromuscular functioning for most patients using the standard criteria, i.e., a decrement of $>10 \%$ and/or a marked increment relative to rest.

\section{Quantitative Myasthenia Gravis Questionnaire}

Results for the raw QMG Total Score and \%Standardized QMG Total Score by treatment group at enrollment are summarized in Table 4 .

The mean \%Standardized QMG Total Score at enrollment among the 20 patients in the Firdapse group (19.9, SD 16.0; equivalent to a QMG Total Score of 7.8), 10 patients in the 3,4-DAP group (23.9, SD 25.0; equivalent to a QMG Total Score of 9.3), and the 9 patients of Other LEMS Treatment group (21.2, SD 21.1; equivalent to a QMG Total Score of 8.3) were similar. The \%Standardized QMG Total Score of the 4 patients receiving Unknown Treatments (11.9 SD, 12.6; equivalent to a QMG Total Score 4.6) was markedly lower than the mean score of the other 3 treatment groups. QMG Total Scores

Table 4 QMG assessment: mean QMG Total Score and \%Standardized QMG Total Score

\begin{tabular}{llllll}
\hline Assessment & Firdapse $(n=\mathbf{3 0})$ & $\mathbf{3 , 4 - D A P}(n=\mathbf{1 5})$ & Other $(\boldsymbol{n}=\mathbf{1 6})$ & Unknown $(\boldsymbol{n}=\mathbf{8})$ & Total $(\boldsymbol{N}=\mathbf{6 9})$ \\
\hline \multicolumn{2}{l}{ QMG Total Score } & & & & \\
$n$ & 21 & 10 & 9 & 4 & 44 \\
Mean (SD) & $7.4(6.3)$ & $9.1(9.7)$ & $5.7(4.7)$ & $4.5(4.8)$ & $7.2(6.8)$ \\
Min-max & $0.0-22.0$ & $0.0-26.0$ & $0.0-13.0$ & $0.0-10.0$ & $0.0-26.0$ \\
\%Standardized Total QMG Score & & & & \\
$n$ & 20 & 10 & 9 & 4 & 43 \\
Mean (SD) & $19.9(16.0)$ & $23.9(25.0)$ & $21.2(21.1)$ & $11.9(12.6)$ & $20.4(18.8)$ \\
Min-max & $0.0-56.4$ & $0.0-66.7$ & $0.0-66.7$ & $0.0-25.6$ & $0.0-66.7$ \\
\hline
\end{tabular}

3,4-DAP 3,4 diaminopyridine, $Q M G$ quantitative myasthenia gravis, $S D$ standard deviation

a \%Standardized Total QMG Score computed as: [raw QMG Total Score/( $N$ of sub-scales with available data $\times$ maximum sub-scale score of 3 )] 
of $>5$ (for complete QMG assessments) have been used as a minimum inclusion criteria in clinical studies evaluating the efficacy of 3,4-DAP. This is to exclude patients who have insufficient disease severity from participation [28].

\section{Reflexes and Muscle Strength}

Patients who had available data at enrollment showed reduced reflexes at the following sites tested: $76.0 \%$ of patients were noted to have a reduced reflex at the knee (38/50), 80\% at the Achilles (10/50), 64\% at the biceps (18/50), and $67 \%$ at the triceps.

Muscle strength for patients who had available data was generally good with the great majority of patients in all 4 treatment groups evaluated as a 4-movement against partial resistance, or as a 5-full strength, in all 12 muscles or muscle groups evaluated. Within these muscle strength response categories, no specific patterns of differences between treatment groups were apparent.

Five patients scored a 2-movement with gravity eliminated, indicating abnormal muscle weakness. Of these 5 patients, 3 were in the Other LEMS treatments group and 2 were in the Firdapse treatment group.

\section{Ataxia}

The majority of patients were assessed as negative for ataxia at enrollment for each of the six standard movement tests administered, both overall and within the four treatment groups. However, there were several exceptions; 4 of the 8 patients (50\%) in the 3,4-DAP group were noted as positive for ataxia during the line walk test, 5 of the 10 patients $(50 \%)$ in the 3,4-DAP group were positive on the heel-to-knee test, and 2 of the 3 patients
$(66.7 \%)$ in the Unknown Treatment group were positive on the line walk test.

\section{Autonomic Nervous System}

The most frequent sign of autonomic abnormality was dry mouth, noted for $51.1 \%$ of patients who had available data, followed by abnormal pupil reflex (24\%), abnormal bladder function (24\%), constipation (21\%), dry eyes $(12 \%)$, orthostatic intolerance $(10 \%)$, and erectile dysfunction/impotence (for $22 \%$ of males). The proportions of patients having autonomic nervous system (ANS) abnormalities by number of signs observed were: None $(3 / 56 ; 5.4 \%), 1$ (6/56; 10.7\%), 2 (20/56; 35.7\%), 3 (18/56; 32.1\%), 4 (5/56; 8.9\%), 5 (3/56; 5.4\%), 6 (1/56; 1.8\%).

\section{Electrocardiography}

ECG data were available for up to 16 (23.2\%) of the 69 enrolled patients, although not all ECG parameters were available for all 16 patients. The mean QT interval for the 9 patients in the Firdapse group [350.7 ms SD 58.2, range 220-418 ms) was similar to the mean QT interval for the 2 patients receiving Other LEMS treatments (357.0 ms SD 4.2, 354-360 ms) and the one patient receiving Unknown Treatment (358.0 ms), but was shorter than the mean value for the 3 patients in the 3,4-DAP group (388.7 ms SD 9.9, range 382-400). The mean associated dose of Firdapse or 3,4-DAP for patients on these treatments was $10.5 \mathrm{mg}$ (SD 6.0, range 5-20 mg).

The mean QTcB interval for the 7 patients in the Firdapse group (423.4 ms SD 36.6) was slightly greater than the mean values for the 3 patients in the 3,4-DAP group (408.7 ms SD 2.3), and greater than both the mean value for the 2 patients receiving Other Treatment (388.0 ms SD 21.2), 
and the value of the single patient receiving Unknown treatment (397.0 ms). The higher mean of the Firdapse group can in part be attributed to 1 patient who had a QTcB of 496 ms. QTcB measurements greater than $440 \mathrm{~ms}$ are considered outside of the normal range.

\section{Pulmonary Function}

Mean values for $\mathrm{FEV}_{1}$ and $\mathrm{FVC}$ showed good congruence to normal reference values across the 3 treatment groups of Firdapse, 3,4-DAP, and Unknown Treatment, with observed mean values by treatment group ranging from $79 \%$ (3,4-DAP) to $97 \%$ (Unknown Treatment) of that predicted for a normal subject. Two patients, both in the 3,4-DAP treatment group and both who never smoked, had $\mathrm{FEV}_{1}$ values of $73 \%$ of predicted and FVC values of $70-71 \%$ of predicted.

\section{Daily Functioning}

The majority of patients who had available data were assessed as having Reduced/Limited functioning for ability to: walk upstairs (37/42, $88.1 \%)$, cycle $(29 / 36,80.6 \%)$, get up from a low chair with and without arm support (25/44, $56.8 \%$ and $32 / 42,76.2 \%$, respectively), get up from sitting on one knee $(29 / 36,80.6 \%)$, get up from squatting $(32 / 40,80.0 \%)$, and climb stairs with and without arm support $(27 / 42,64.3 \%$ and 31/42, 73.8\%, respectively). Reduced/ Limited functioning was also noted for majority of the assessed patients for the comparatively easier tasks of: walking on toes and heels (24/43, 55.8\% and 24/42, 57.1\%, respectively), and getting up from a high chair without arm support (25/41, 61.0\%). Reduced ability was assessed for 19/41 (46.3\%) of the patients getting up from a high chair with arm support.

\section{EQ-5D Health Status}

Among patients who had available data, the majority of patients $(26 / 36,72.2 \%)$ responded to the mobility scale as having "2-some problems" with walking. Three patients (1 receiving 3,4-DAP, and 2 receiving Firdapse) responded "3-confined to bed". Over half of patients $(20 / 36,55.6 \%)$ reported no problems with self-care. The remainder reported either having some problems $(13 / 36,36.1 \%)$ and 3 patients (2 receiving 3,4-DAP and 1 receiving Firdapse) reported being unable to wash or dress $(3 / 36,8.3 \%)$. Similarly, the majority of patients $(21 / 36,58.3 \%)$ reported having some problems with performing their usual activities, and 5/36 patients $(13.9 \%)$ were unable to perform their usual activities. Over half of patients (21/36, $58.3 \%$ ) responded as having "moderate pain or discomfort", while the remainder (15/36; $41.7 \%)$ stated they had no pain or discomfort. Fewer patients (14/36, 38.9\%) reported moderate problems with anxiety and/or depression.

When asked to rate "How good or bad your own health is" on a 100-point visual analog scale (VAS), the mean responses from patients receiving Unknown LEMS treatments, Firdapse, and 3,4-DAP treatment groups ranged from low to moderately low. Mean values were: Unknown Treatment (28.7, SD 22.03, $n=3$ ), Firdapse (34.8, SD 34.30, $n=15)$, and 3,4-DAP (49.5, SD 26.31, $n=11)$. In contrast, the mean VAS health score for Other Treatments was considerably higher (66.0, SD 35.95, $n=5)$.

\section{DISCUSSION}

LEMS is a rare disease and available information on the associated population statistics, clinical course and manifestations, treatment practices, 
and outcomes is sparse to non-existent. Eligibility for participation in the registry was confirmed by EMG findings that met standard criteria as defined by percent increment and decrement in CMAP amplitude, by elevated $\mathrm{Ca}_{\mathrm{v}} 2.1 \mathrm{P} / \mathrm{Q}$-type VGCC antibody titers, and by clinical evaluation. While the $\mathrm{Ca}_{\mathrm{v}} 2.1 \mathrm{P} / \mathrm{Q}$-type autoantibody assay is the most relevant test in evaluating the underlying etiology of neuromuscular signs in the suspected LEMS patient, it should be pointed out that an N-type (Cav2.2) autoantibody assay may be more reflective of signs of dysautonomia since many autonomic nerve terminals release ACh from N-type, rather than P/Q-type, Ca channels. Although there is presently a lack of data characterizing the relationship between N-type autoantibody titers and the incidence and severity of dysautonomia in patients with confirmed LEMS, this is an interesting topic and merits further investigation.

Abnormal electrophysiological findings, along with the presence of elevated VGCC autoantibody titers, confirm a diagnosis of LEMS. The degree of abnormality in CMAP amplitude indicates the severity of neuromuscular block [19] with CMAP amplitude decreasing proportionally with increasing severity of LEMS disease. Data yielded by electrophysiological evaluation represent an important means of establishing baseline severity, permits long-term monitoring of disease progression and staging, and allows the clinician to objectively communicate and report on clinical status for purposes such as continuity of care and research. Electrophysiological testing protocols must be adhered to, however, to achieve consistency and reliability of results across clinical centers. CMAP may be used to periodically monitor the clinical effectiveness of treatment over the long-term. Test kits for assay of $\mathrm{Ca}_{\mathrm{v}} 2.1$
P/Q-type VGCC autoantibody titers are commercially available.

Males and females were equally represented in this study population and there was a wide range of ages at enrollment (27-84 years). Most patients never smoked or smoked previously but had since quit. Review of the overall clinical findings at enrollment presented a picture of generally mild-to-moderate neuromuscular and autonomic impairment for most participants in the registry. The majority of patients were assessed as having reduced or limited functioning for activities associated with daily functioning, such as the ability to walk upstairs, cycle, arise from a low chair with and without arm support, arise from sitting on one knee or squatting, and climbing stairs with and without arm support. Fewer patients, although still a majority, were assessed as having reduced or limited functioning for the comparatively easier tasks of walking on toes and heels and arise from a high chair with and without arm support. These findings are consistent with responses from the EQ-5D health questionnaire which indicated the majority of patients have some problems with mobility and with performing their usual activities. Patients with severe impairment (e.g., confinement to bed, pulmonary function parameters $<80 \%$ of normal, unable to care for self, multiple tests positive for ataxia) were relatively few, approximately $5(7.2 \%)$ in this population. These above findings are also consistent with that reported elsewhere in the literature-“... most patients suffer multiple symptoms which are frequently severe and troublesome, and almost all are restricted in ADL (activities of daily living) with poor health status. There is high utilization of healthcare resources from diagnosis to ongoing treatment" [12].

Consistent with the profile of only mild-to-moderate deficits reported for activities 
associated with daily functioning, muscle strength was generally good for most patients, but most also had reduced deep tendon reflexes at all 4 anatomic locations tested and 38\% demonstrated ataxia during the line walk test. There were fewer patients positive for the remaining tests of ataxia or signs of autonomic dysfunction (except for dry mouth which was a common finding). Three quarters of people with LEMS have disruption of ANS functioning [29] and the frequency of cerebellar ataxia (CA) in patients with LEMS is higher than expected by chance. When it occurs, it is usually associated with carcinoma [30]. Cerebellar degeneration leading to ataxia can be paraneoplastic in origin, a non-metastatic complication of carcinoma. It is thought that this process is mediated by antibody response against tumor antigens, especially against the $\mathrm{Ca}_{\mathrm{v}} 2.1$ P/Q-type VGCCs which are highly expressed in the cerebellum, leading to cerebellar degeneration [31]. It is recommended that patients with LEMS be periodically assessed and monitored for the onset of ataxia which can indicate the presence of a degenerative cerebellar process of paraneoplastic origin. Approximately 50-60\% of patients in the registry have an underlying tumor (paraneoplastic form), but LEMS can also occur in the absence of cancer (idiopathic form). The prevalence of the two forms is similar but the incidence of the paraneoplastic form is much higher [32]. This latter form is most commonly associated with SCLC but can also result from other malignancies [8]. Because only $50-70 \%$ of patients with LEMS have an identifiable cancer and because LEMS remains undiagnosed in many cancer patients, the true total prevalence of LEMS may be considerably higher.

No consistent pattern of QT interval differences was apparent in comparing mean values between treatment groups. ECG data were limited, however. Overall, the observed QT intervals were within the normal ranges for the overall registry population. The small observed differences in the mean QTCB interval between treatment groups was largely due to the outlying values contributed by one patient in the Firdapse treatment group who had a QTcB interval of $496 \mathrm{~ms}$ and who also had a prolonged PQ interval of $212 \mathrm{~ms}$.

Mean spirometry values for $\mathrm{FEV}_{1}$ and FVC for most patients showed good agreement with normal reference values with observed mean values ranging from $79 \%$ to $97 \%$ of that predicted for a normal subject based on patient age, gender, and height. There were 2 patients with lower than expected pulmonary functioning at baseline.

Psychologically, 39\% of patients reported having moderate problems with anxiety and/ or depression. In terms of pain and discomfort, overall, over half $(58.3 \%)$ reported moderate pain or discomfort. Two patients (both in the Other LEMS treatments group) reported extreme pain or discomfort.

\section{Treatment Characteristics}

Since most patients had been receiving LEMS treatments to improve neuromuscular and autonomic functioning for at least a short time by enrollment, it is likely that the picture of mild-to-moderate impairment for most patients had already reflected some degree of improvement resulting from initiation of treatment. Forty-five of the 69 patients in the registry $(65 \%)$ were on either Firdapse $(n=30)$ or 3,4-DAP base $(n=15)$ at registry enrollment. Forty patients (58\%) were on Firdapse at some point, either before, during or after enrollment. Most of the 30 patients receiving Firdapse at enrollment had been receiving it for a period of 
between 2 days and up to 14.8 years, and at least 16 of those patients had been on Firdapse for greater than 1 year. Patients were on total daily doses of $10-80 \mathrm{mg}$. These dose and treatment duration characteristics were approximately similar for patients prescribed 3,4-DAP as the base form.

Immunomodulators (immunosuppressants) were the most frequently prescribed LEMS-specific treatment other than Firdapse or 3,4-DAP base: 12 patients (17.4\%) received azathioprine, 2 patients $(2.9 \%)$ received cyclophosphamide, 4 patients $(5.8 \%)$ received MMF, 2 patients (2.9\%) received cyclosporine A, 19 patients $(27.5 \%)$ received prednisone, 1 patient $(1.4 \%)$ received methylprednisolone, and 10 patients $(14.5 \%)$ received IVIg therapy. Twenty-two patients (31.9\%) received pyridostigmine.

Firdapse is currently the only approved 3,4 DAP compound for the symptomatic treatment of LEMS in adults in the EU. 3,4-DAP as the free base is not approved as a medicinal product, but is available for LEMS patients from compounding pharmacies in several EU countries. However, concern remains over the form of amifampridine prescribed since considerable variability has been observed in the active pharmaceutical ingredient (API) content quantified from laboratory analyses of samples of compounded 3,4 DAP base [33]. All 10 samples from 1 compounded product had only a fraction of the API content of the label claim [35.0\%, 51.7\% relative standard deviation (RSD)]. In addition, no compounded 3,4-DAP product met Good Manufacturing Practice standards of having $95-105 \%$ content of the claimed label content; one sample was observed to have $90-110 \%$, and 4 others achieved $80-120 \%$ of declared content for all 10 samples [33]. No degradation products were found in any compounded sample tested.
Treatment of LEMS is generally classified into 3 distinct but often complementary approaches. Symptomatic treatments, agents administered to amplify or enhance impaired neuromuscular transmission, are further classified according to the functional mechanism of action that improves the neuromuscular transmission. These are generally comprised of parasympathomimetic agents that act either indirectly, by increasing the concentration of ACh in the NMJ through inhibition of the AChE (e.g., pyridostigmine), or act directly, by increasing the release of ACh into the NMJ. This latter class of agents consists of potassium channel blockers such as guanidine, recommended originally by Lambert to treat LEMS [34], and the aminopyridines, namely 4-aminopyridine (4-AP) and 3,4-DAP (or amifampridine [35]) or its phosphate salt, 3,4-DAP phosphate (amifampridine phosphate, Firdapse). However, guanidine is associated with hazardous side effects and its use is limited in clinical practice.

The aminopyridines act by blocking voltage-gated potassium channels, thereby prolonging the action potential and increasing calcium ion $\left(\mathrm{Ca}^{2+}\right)$ influx to the nerve terminal. Consequently, ACh release is increased, improving neuromuscular transmission and muscle function.

Immunomodulating treatment putatively seeks to reduce the tumor antigen-autoantibody response, presumably the lowering of antibody activity directed against $\mathrm{Ca}_{\mathrm{v}} 2.1 \mathrm{P} / \mathrm{Q}$-type VGCCs that mediate ACh release into the NMJ. However, even in patients in whom carcinoma has been ruled out, immunosuppression has been shown to have benefit [36]. Immunosuppressive agents such as oral corticosteroids (e.g., prednisolone), azathioprine, MMF, rituximab, or other immunosuppressive agents are frequently 
employed. The objective of therapeutic plasma exchange is to reduce circulating antibody concentrations. Although recommended by the National Institute of Health and supported by numerous case reports, no prospective, comparator-controlled evaluations have been published to date. Therefore, long-term use is not supported by available data and it is not part of the current guidelines in the clinical neurology practice. In the hands of expert physicians, however, it may be safely used.

Anticancer treatment is indicated for patients who have the paraneoplastic form of LEMS. An algorithm to organize the sequence of each of the above treatment approaches was first proposed in 1998 [37], but until recently only little has existed in the way of formalized, evidence-based guidelines. This is almost certainly owing to the rarity of the disease and only a gradual accumulation of clinical evidence and a slow evolution in optimizing treatment approaches. Several consensus guidelines and review articles on the treatment and management of the neuromuscular transmission disorders, including LEMS, have since emerged in recent years, however.

Five randomized, controlled trials consisting of a total of 54 participants with LEMS and who were treated with 3,4-DAP or placebo, and 9 patients who were treated with IVIg or placebo, were identified and included in the review. The authors concluded that there was "...moderate to high-quality evidence from randomised controlled trials that either 3,4-DAP or IVIg improved muscle strength scores and compound muscle action potential amplitudes in people with LEMS." The authors recommended using QMG scores as the primary outcome and change in CMAP as the secondary outcome.

A similar report reviewing substantially the same and other data published in 2011 [38] reached essentially equivalent conclusions regarding the use of 3,4-DAP as a first-line symptomatic treatment in combination with long-term immunosuppression, with or without antitumor therapy as indicated. The author notes that plasma exchange and short-term use of IVIg have shown acute benefit but are not recommended for long-term, intermittent use.

Treatment recommendations from another review [39] echo virtually the same algorithm of approaches to treatment, i.e., first-line symptomatic treatment with 3,4-DAP/3,4-DAP phosphate, followed by immunosuppressive drug therapy, additional immunomodulation with IVIg, treatment of underlying SCLC if present, and possibly adding on plasma exchange as an acute adjunct.

QMG symptoms were assessed by standardized protocol $[20,21]$. The mean \%Standardized QMG Total Score at enrollment among the 20 patients in the Firdapse group (19.9, SD 16.0; equivalent to a QMG Total Score of 7.8), 10 patients in the 3,4 DAP group (23.9, SD 25.0; equivalent to a QMG Total Score of 9.3), and the 9 patients of Other LEMS Treatment group (21.2, SD 21.1; equivalent to a QMG Total Score of 8.3) were similar. The \%Standardized QMG Total Score of the 4 patients receiving Unknown Treatments (11.9 SD, 12.6; equivalent to a QMG Total Score 4.6) was markedly lower than the mean score of the other 3 treatment groups. QMG Total Scores of $>5$ (for complete QMG assessments) have been used as a minimum inclusion criteria in clinical studies evaluating the efficacy of 3,4-DAP.

\section{CONCLUSIONS}

The LEMS EU registry will continue to enroll patients and periodically report on the clinical outcomes of this patient population. The 
findings of this registry will contribute to a better understanding of LEMS and its natural history, as well as the diagnostic and therapeutic challenges facing patients and the healthcare professionals who care for them.

\section{ACKNOWLEDGMENTS}

Sponsorship for this study and article processing charges were funded by BioMarin Pharmaceutical Inc. (Novato, CA, USA). All named authors meet the International Committee of Medical Journal Editors (ICMJE) criteria for authorship for this manuscript, take responsibility for the integrity of the work as a whole, and have given final approval to the version to be published. The authors would like to acknowledge and thank the following registry sub-investigators at each of their respective sites: Drs. Barbara Pasanisi at Prof. Dr. Mantegazza's site; Drs. Siegfried Kohler at Prof. Meisel's site; Mrs. Morgane Gaboreau at Prof. Dr. Le Masson and Dr. Wael Marouf at Prof. Joern P. Sieb's site. The authors would further like to acknowledge and thank the following additional Principle Investigators (PI), Sub-Investigators (SI), study coordinators (SC), and their respective host institutions: Prof. Amelia Evoli (PI) and Dr. Paolo Alboini (SI) Universita Cattolica del Sacro Cuore, Dipartimento di Neuroscienze, Policlinico Universitario Agostino Gemelli, Rome, Italy; Prof. Carmelo Rodolico (PI), Dr. Simona Portaro (SI), Policlinico Universitario G. Martino, Messina, Italy; Dr. Adrien Didelot (PI), Centre Hospitalier Lyon Sud Service d'Explorations Fonctionnelles Neurologiques, Lyon, France; Dr. Alfredo Romani (PI) and Dr. Laura Piccolo (SI), Istituto Neurologico Nazionale C. Mondino, Pavia, Italy; Dr. Andreas Schröter (PI), Klinikum Bremen Ost, Klinik fur Neurologie, Bremen, Germany; Prof.
Jorn Schattschneider (PI), Neurologie Ev.-Luth. Diakonissenanstalt zu Flensburg Zentrum für Gesundheit und Diakonie Knuthstr., Flensburg, Germany; Ms. Elisabeth Llinares (SC), Centre Hospitalier Universitaires Nimes, Hospital Caremeau, Neurology Department, Nimes, France; Dr. Amar Smail (PI) and Mrs. Delphine Quignion (SC), Department of Internal Medicine, Centre Hospitalier, Universitaires de Amiens, Hôpital Nord, Place Victor Pauchet, Amiens, France; Dr. Vivien Pautot (PI), Centre Hospitalier Universitaires de Angers, Fonctionnelles Department, Angers, France; Dr. Peter Trillenberg (PI) Universitätsklinikum Schleswig-Holstein Campus Lübeck Klinik für Neurologi.e. Lübeck, Germany; Dr. Karl Knop (PI), Neurologie Neuer Wall, Hamburg, Germany; and Dr. Marina Frasquet Carrera (SI), Department of Medicine, University of Valencia, Hospital Universitari i Politecnic La Fe, Valencia, Spain. The authors would like to thank and acknowledge Lawrence Linn of Clinical Science and Medical Writing Consulting Services (San Francisco Bay Area, California, USA) for his assistance in the preparation of this manuscript. Support for the editorial assistance was BioMarin Pharmaceutical Inc.

Disclosures. Renato Mantegazza has received financial compensation for his participation as an Investigator in the LEMS Registry, as a member of the LEMS Registry Advisory Board, and as a Consultants to BioMarin Europe Ltd. Andreas Meisel has received financial compensation for his participation as an Investigator in the LEMS Registry, as a member of the LEMS Registry Advisory Board, and as a Consultants to BioMarin Europe Ltd. Joern P. Sieb has received financial compensation for his participation as an Investigator in the LEMS Registry, as a member of the LEMS Registry 
Advisory Board, and as a Consultants to BioMarin Europe Ltd. Gwendal Le Masson has received financial compensation for his participation as an Investigator in the LEMS Registry, as a member of the LEMS Registry Advisory Board, and as a Consultants to BioMarin Europe Ltd. Claude Desnuelle has received financial compensation for his participation as an Investigator in the LEMS Registry, as a member of the LEMS Registry Advisory Board, and as a Consultants to BioMarin Europe Ltd. Mirko Essing is an employee of BioMarin Europe Ltd.

Compliance with ethics guidelines. All registry procedures that were and are being conducted in accordance with the ethical standards of the responsible committee on human experimentation (institutional and national) and with the Helsinki Declaration of 1964, as revised in 2013. Informed consent was obtained from all patients included in the study. The LEMS registry protocol, patient information sheet, and informed consent form have been submitted to and approved by an ethics committee (EC), subject to applicable local laws at each center where the registry is being conducted. In countries participating in the LEMS registry where approvals other than an EC approval were required, all applicable requirements were met and approved. Applicable approvals were completed and in place prior to site initiation and patient enrollment. All documents are on file at the registry's Sponsor, BioMarin Europe Ltd. (London, UK).

Open Access. This article is distributed under the terms of the Creative Commons Attribution-NonCommercial 4.0 International License (http://creativecommons.org/licenses/ by-nc/4.0/), which permits any noncommercial use, distribution, and reproduction in any medium, provided you give appropriate credit to the original author(s) and the source, provide a link to the Creative Commons license, and indicate if changes were made.

\section{REFERENCES}

1. Bekircan-Kurt CE, Çiftçi ED, Kurne AT, Anlar B. Voltage gated calcium channel antibody-related neurological diseases. World J Clin Cases. 2015;3(3):293-300.

2. Yamakage M, Namiki A. Calcium channels-basic aspects of their structure, function and gene encoding; anesthetic action on the channels-a review. Can J Anesth. 2002;49(2):151-64.

3. Lennon VA, Kryzer TJ, Griesmann GE, et al. Calcium-channel antibodies in the Lambert-Eaton Syndrome and other paraneoplastic syndromes. N Engl J Med. 1995;332:1467-74.

4. Seneviratne $U$, de Silva R. Lambert-Eaton myasthenic syndrome. Postgrad Med J. 1999;75:516-20.

5. Newsom-Davis J. Lambert-Eaton myasthenic syndrome. Rev Neurol (Paris). 2004;160(2):177-80.

6. Titulaer M. Lambert-Eaton myasthenic syndrome [Internet]. 2013 [updated 2013 November]. Available from: http://www.orpha.net.

7. Wirtz PW, Nijnuis MG, Sotodeh M, et al. The epidemiology of myasthenia gravis, Lambert-Eaton myasthenic syndrome and their associated tumours in the northern part of the province of South Holland. J Neurol. 2003;250(6):698-701.

8. O'Neill JH, Murray NMF, Newsom-Davis J. The Lambert-Eaton Myasthenic syndrome. A review of 50 cases. Brain. 1988;111(Pt.3):577-96.

9. Gutmann L, Phillips LH, Gutmann L. Trends in the association of Lambert-Eaton myasthenic syndrome with carcinoma. Neurology. 1992;42(4):848-50.

10. Payne M, Bradbury P, Lang B, et al. Prospective study into the incidence of Lambert Eaton myasthenic syndrome in small cell lung cancer. J Thorac Oncol. 2010;5(1):34-8.

11. Stickler DE. Lambert-Eaton Myasthenic Sydrome (LEMS) [Internet]. 2013 [updated 2013 May3]. 
Available from http://emedicine.medscape.com/ article/1170810-overview.

12. Harms L, Peter Sieb J-P, Williams AE, et al. Long-term disease history, clinical symptoms, health status, and healthcare utilization in patients suffering from Lambert Eaton myasthenic syndrome: results of a patient interview survey in Germany. J Med Econ. 2012;15(3):521-30.

13. Sanders DB. Lambert-Eaton myasthenic syndrome: diagnosis and treatment. Ann N Y Acad Sci. 2003;998:500-8.

14. Vedeler CA, Antoine JC, Giometto B, et al. Management of paraneoplastic neurological syndromes: report of an EFNS Task Force. Eur J Neurol. 2006;13(7):682-90.

15. Skeie GO, Apostolskib S, Evolic A, et al. Guidelines for treatment of autoimmune neuromuscular transmission disorders. Eur J Neurol. 2010;17:893-902.

16. Vedeler CA, Antoine JC, Giometto B, et al. Paraneoplastic neurological syndromes. In: Gilhus NE, Barnes MP, Brainin M, editors. European handbook of neurological management, vol. 1. 2nd ed. Oxford (UK): Wiley-Blackwell; 2011.

17. AAEM Quality Assurance Committee. Literature review of the usefulness of repetitive nerve stimulation and single fiber EMG in the electrodiagnostic evaluation of patients with suspected myasthenia gravis or Lambert-Eaton myasthenic syndrome. Muscle Nerve. 2001;24(9):1239-47.

18. AAEM Quality Assurance Committee. Practice parameter for repetitive nerve stimulation and single fiber EMG evaluation of adults with suspected myasthenia gravis or Lambert-Eaton myasthenic syndrome: summary statement. Muscle Nerve. 2001;24:1236-8.

19. Wirtz PW, Verschuren JJ, van Dijk JG, et al. Efficacy of 3,4 diaminopyridine and pyridostigmine in the treatment of Lambert-Eaton myasthenic syndrome: a randomized double-blind placebo controlled cross over study. Clin Pharmacol Ther. $2009 ; 86: 44-8$.

20. Barohn RJ, McIntire D, Herbelin L, et al. Reliability testing of the quantitative myasthenia gravis score. Ann N Y Acad Sci. 1998;841:769-72.

21. Jablecki C. Lambert-Eaton myasthenic syndrome. Muscle Nerve. 1984;7(3):250-7.

22. Boerma CE, Rommes JH, van Leeuwen RB, Bakker J. Cardiac arrest following a iatrogenic by 3,4-diaminopyridine intoxication in a patient with Lambert-Eaton myasthenic syndrome. J Toxicol Clin Toxicol. 1995;33:249-51.

23. Lundh H, Nilsson O, Rosen I, Johansson S. Practical aspects of 3,4-diaminopyridine treatment of the Lambert-Eaton myasthenic syndrome. Acta Neurol Scand. 1993;88:136-40.

24. Wilcox PG, Morrison NJ, Anzarut AR, Pardy RL. Lambert-Eaton myasthenic syndrome involving the diaphragm. Chest. 1988;93:604-6.

25. Hankinson JL, Odencrantz JR, Fedan KB. Spirometric reference values from a sample of the general US population. Am J Respir Crit Care Med. 1999;159:179-87.

26. The EuroQol Group. EuroQol-a new facility for the measurement of health-related quality of life. Health Policy. 1990;16(3):199-208.

27. Motomura M, Johnston I, Lang B, Vincent A, Newsom-Davis J. An improved diagnostic assay for Lambert-Eaton myasthenic syndrome. J Neurol Neurosurg Psychiatry. 1995;58:85-7.

28. Sanders DB, Massey JM, Sanders LL, Edwards LJ. A randomized trial of 3,4-diaminopyridine in Lambert-Eaton myasthenic syndrome. Neurology. 2000;54(3):603-7.

29. Mareska M, Gutmann L. Lambert-Eaton myasthenic syndrome. Semin Neurol. 2004;24(2):149-53.

30. Clouston PD, Saper CB, Arbizu T, et al. Paraneoplastic cerebellar degeneration. III. Cerebellar degeneration, cancer, and the Lambert-Eaton myasthenic syndrome. Neurology. 1992;42:1944-50.

31. Honnorat J. Understanding the physiopathology of paraneoplastic and genetic cerebellar ataxia. J Neurol Neurosurg Psychiatry. 2006;77(12):1299-300.

32. Newsom-Davis J. Therapy in myasthenia gravis and Lambert-Eaton myasthenic syndrome. Semin Neurol. 2003;23(2):191-8.

33. Green DM, Jones AC, Brain KR. Content variability of active drug substance in compounded oral 3,4-diaminopyridine products. J Clin Pharm Ther. 2012;37:53-7.

34. Lambert EH. Defects of neuromuscular transmission in syndromes other than myasthenia gravis. Ann N Y Acad Sci. 1966;135:367-84.

35. McEvoy KM, Windebank AJ, Daube JR, Low PA. 3,4-Diaminopyridine in the treatment of 
Lambert-Eaton myasthenic syndrome. N Engl J Med. 1989;321(23):1567-71.

36. Maddison P, Lang B, Mills K, Newsom-Davis J. Long term outcome in Lambert-Eaton myasthenic syndrome without lung cancer. J Neurol Neurosurg Psychiatry. 2001;70:212-7.

37. Newsom-Davis J. A treatment algorithm for Lambert-Eaton myasthenic syndrome. Ann N Y Acad Sci. 1998;841:817-22.
38. Maddison P. Treatment of Lambert-Eaton myasthenic syndrome. Ann N Y Acad. 2012;1275:78-84.

39. Gilhus NE. Lambert-Eaton myasthenic syndrome; pathogenesis, diagnosis, and therapy. Autoimmune Dis. 2011;2011:1-5. doi:10.4061/2011/973808. 